\title{
ETUDE SUR MODĖLE RÉDUIT DE LA DÉRIVATION PROVISOIRE DU ZÉZĖRE A CASTELO DO BODE (PORTUGAL)
}

\section{SMALL-SCALE MODEL STUDIES OF A TEMPORARY DIVERSION OF CASTELO DO BODE}

\author{
par J. RUEFF \\ Ingénieur civil des Ponts et Chaussées
}

(English synopsis p. 775)

\section{I. - PROBLÈME GÉNÉRAL}

Malgré leur caractère provisoire, les ouvrages de dérivation constituent assez souvent une partie importante de l'ensemble des travaux d'aménagement d'une chute.

Leur coût, loin d'être négligeable, et la néces.. sité de se prémunir contre les préjudices et retards qu'occasionneraient les inondations du chantier en cas d'insuffisance de la galerie, justifient bien souvent à eux seuls une étude assez approfondie.

Enfin, elles peuvent parfois être adaptées pour une utilisation définitive: prise d'eau. vidange de fond, évacuation des crues.

Le débit à dériver est fixé en fonction des conditions hydrologiques observées au cours de mesures antérieures; la longueur de la galerie est imposée par les dispositions d'ensemble du chantier.

Le problème du dimensionnement le plus économique des ouvrages de dérivation consiste alors à rechercher un compromis entre la section de la galerie et la hauteur du batardeau amont.

La hauteur $\mathrm{H}$ de retenue de ce batardeau est la somme:

du niveau aval h pour la crue considérée, des pertes de charge $P_{1}$ à la sortie de la galerie,

des pertes de charge $P_{2}$ dans la galerie proprement dite, des pertes de charge $P_{3}$ à l'entrée de la galerie,

$$
\mathrm{H}=\mathrm{h}+\mathrm{P}_{1}+\mathrm{P}_{2}+\mathrm{P}_{3}
$$

- $h$ découle directement de la première donnée du projet — le débit - la loi hauteur-débit de la rivière étant connue.

- $P_{2}$ est fonction de la forme et de la rugosité de la galerie qui peuvent ellesmêmes dépendre indirectement de la nature du terrain. Pour une section de galerie donnée en bon rocher, on peut hésiter entre une galerie non revêtue, plus économique, mais donnant une plus grande perte de charge, et une galerie revêtue.

La comparaison de l'augmentation de prix du batardeau et de la diminu. tion de prix de la galerie permettra de choisir immédiatement la solution la plus avantageuse.

- $P_{1}$ et $P_{3}$, pertes de charge à l'entrée et à la sortie de la galerie, vont constituer l'objet principal des essais sur modèle réduit.

La perte de charge $P_{3}$ à l'entonnement est assez intuitive; on conçoit qu'elle va varier avec les dispositions et les formes de l'entrée de la galerie. Avec des formes simples, mais bien étudiées, on peut normalement réduire cette perte 


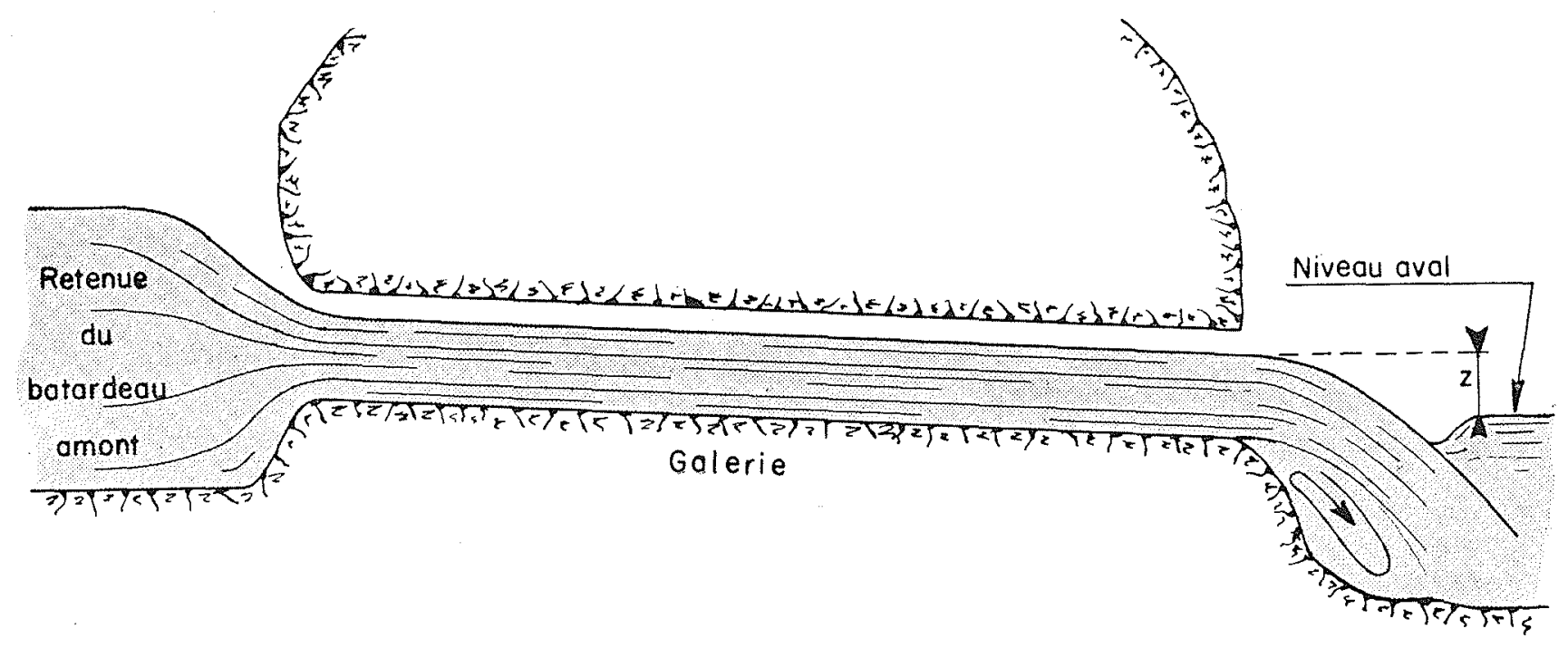

fig. 1

à $10 \%$ environ de l'énergie cinétique, soit $\checkmark$

$0.1 \frac{}{2 g} ; V$ étant la vitesse moyenne dans la section courante de la galerie.

La façon dont intervient la perte de charge $P_{i}$ est moins intuitive, aussi insisterons-nous un peu plus sur elle.

Considérons la galerie schématisée sur la figure 1 .

Le niveau de l'eau à la sortie de la galerie est situé à $z$ mètres au-dessus du niveau aval.

Si l'on abaise l'ensemble de la galerie de $z$ mètres sans changer sa pente et sa section, le niveau à la sortie sera à la cote du niveau aval. Les conditions d'écoulement dans la galerie n'auront pas changé ; le niveau amont, et par suite la hauteur du batardeau correspondant, auront baissé de $z$ mètres, le coût de la galerie étant resté le même.

Dans ces conditions, l'énergie à la sortie de la galerie se trouve être encore très supérieure à l'énergie dans la rivière, si bien que l'on dispose d'une certaine marge pour continuer à baisser la galerie, tout en «chassant» le niveau aval, ce qui correspond à une nouvelle réduction de la hauteur du batardeau amont.

Naturellement, la quantité dont on pourra baisser le niveau à la sortie de la galerie en dessous du niveau aval de la rivière (autrement dit la récupération d'énergie cinétique) dépend essentiellement des formes de la tête aval.
Pour établir le projet d'une dérivation, on sera donc conduit admettre à priori une certaine récupération d'énergie cinétique à la sortie de la galerie et l'on détermine en conséquence le calage en altitude de celle-ci; on cherchera ensuite les formes à donner à la tête aval pour obtenir effectivement cette récupération.

Les procédés habituellement employés à cette fin sont la création d'un ressaut et la récupération par divergent en charge.

Pour la galerie de Castelo do Bode, nous avons employé un procédé un peu différent, la récupération en régime torrentiel. Ce procédé peut d'ailleurs être combiné avec le ressaut pour obtenir une récupération très poussée, tout en conservant une tête aval de dimensions assez restreintes.

\section{1. - SITUATION \\ ET CARACTÉRISTIQUES PRINCIPALES DE LA GALERIE}

Le barrage de Castelo do Bode, actuellement en construction, est situé sur le rio Zêzere, affluent de la rive droite du Tage.

Comme la plupart des rivières portugaises, le Zêzere a des étiages très marqués et des crues très importantes, aussi le débit à dériver a-t-il été fixé à $2.000 \mathrm{~m}^{3} / \mathrm{sec}$.

Pour ce débit, plusieurs solutions ont été étudiées successivement, correspondant aux divers 


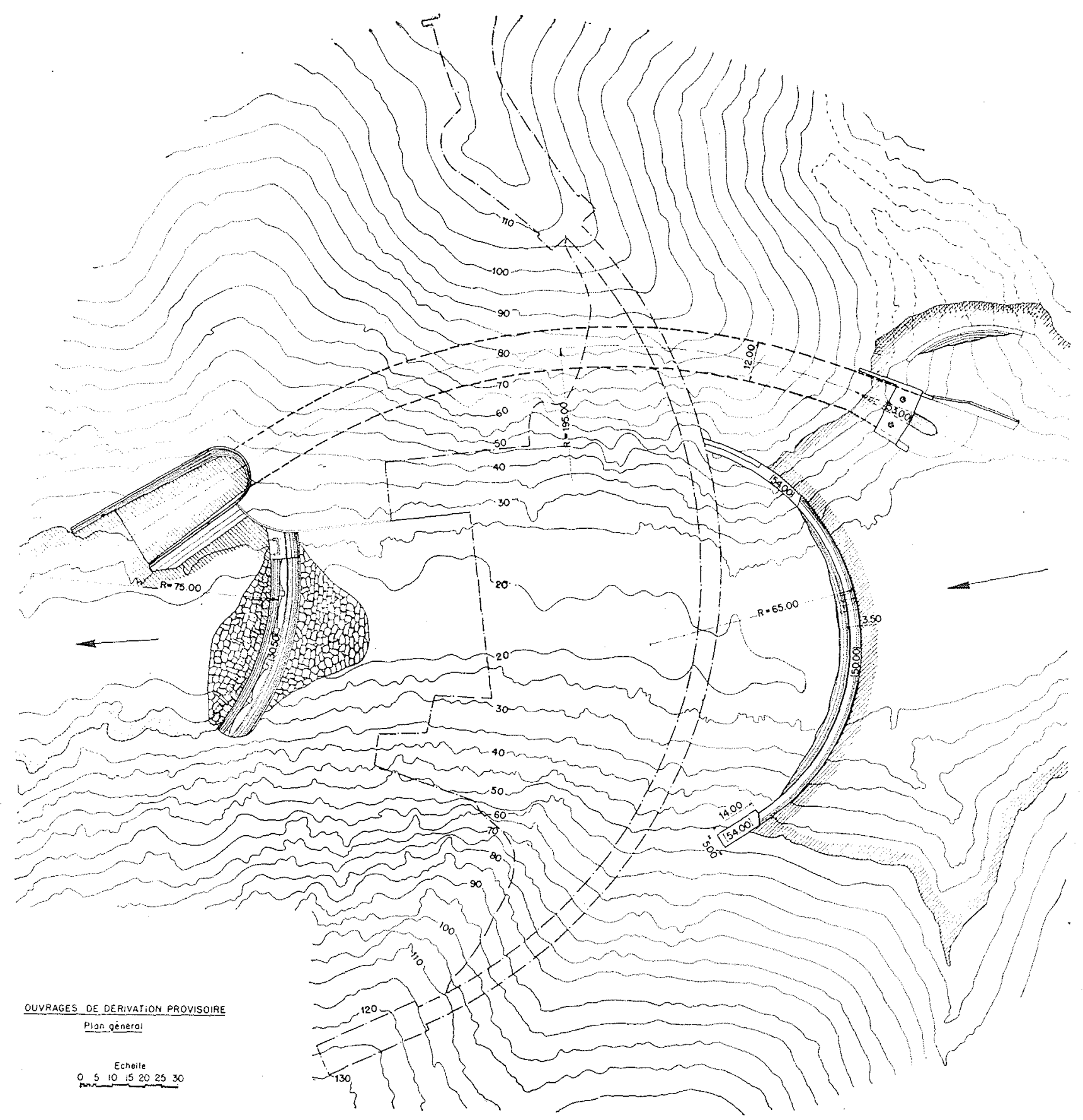

fig. 2

Le chantier de l'usine-barrage de Castelo do Bode

projets d'aménagement de l'ensemble du chantier.

Les deux premiers projets, comportant deux galeries (une sur chaque rive) de $78,5 \mathrm{~m}^{2}$ de section chacune, ont été étudiés sur modèle réduit au Laboratoire Dauphinois d'Hydraulique des Etablissements Neyrpic, à Grenoble.

Le troisième projet prévoyait une galerie unique de $99 \mathrm{~m}^{2}$ de section sur la rive droite, le batardeau-voûte amont étant d'autre part légè. rement surélevé. 


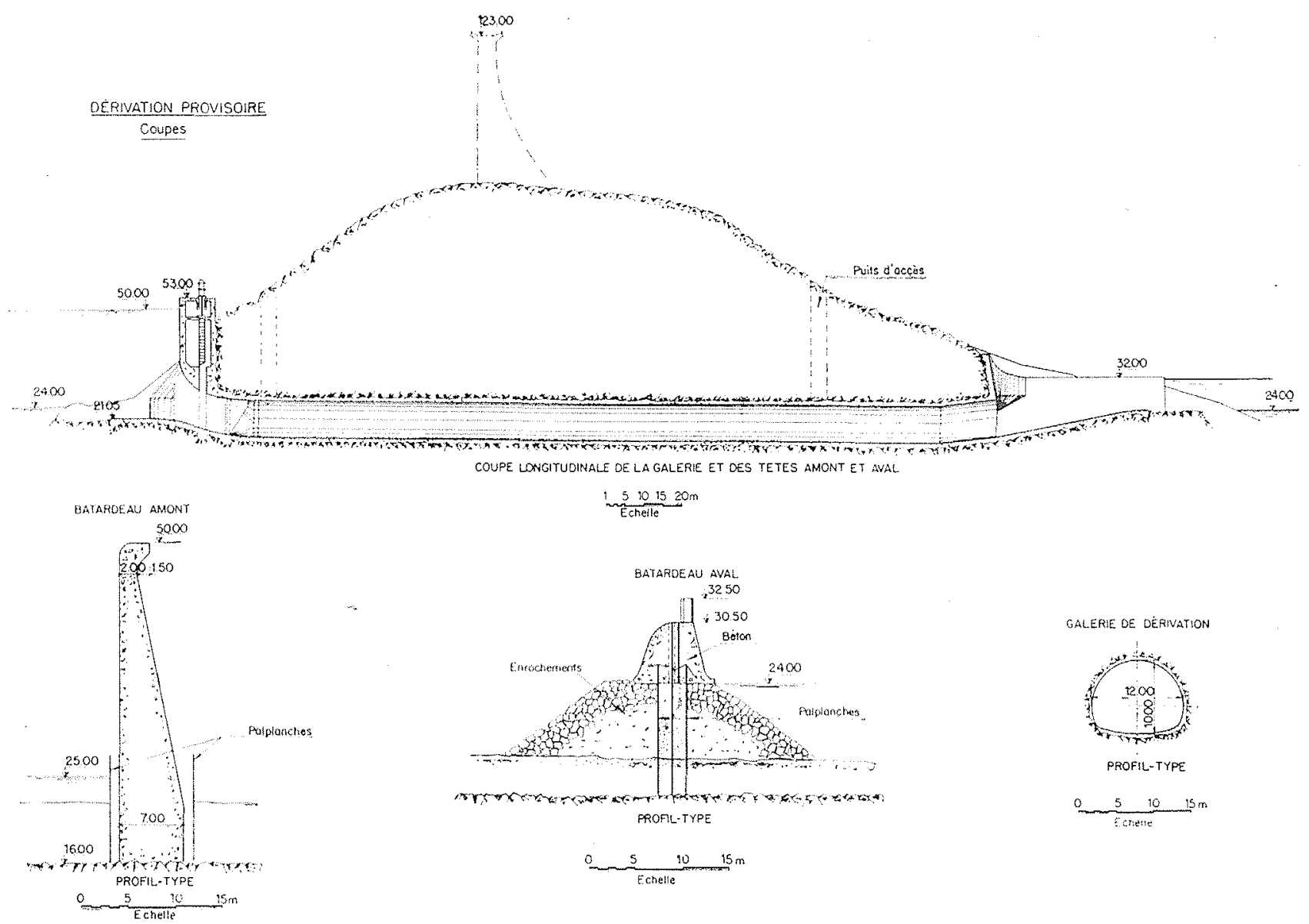

fig. 2.1

Usmobarate de Casteto do Bode. - Les ouvages de derivation provisote : batardeax ef galerie

$\mathrm{Ce}$ projet, dont les dispositions principales sont représentées sur la figure 2 et $2 \mathrm{~A}$ a fait l'objet d'études sur modèle réduit réalisées au Laboratoire d'Hydraulique construit à Amadora (Portugal) sous l'orientation technique des Etablissements Neyrpic.

\section{III. - CARACTÉRISTIQUES DU MODÈLE RÉDUIT}

Le modèle réduit (fig. 3) représente, à l'échelle $1 / 50^{\circ}$, le cours du Zêzere de $250 \mathrm{~m}$. à l'amont du barrage, jusqu'à $760 \mathrm{~m}$. à l'aval, ainsi que les ouvrages de dérivation. Il est muni de l'appareil. lage de mesure nécessaire aux essais. Le débit, réglé à la demande par un système de vannage. est préalablement mesuré, avant son entrée dans le modèle, au moyen d'un déversoir triangulaire à $90^{\circ}$. Le niveau, dans la partie aval du modèle, est réglé automatiquement en fonction du débit par un déversoir mis au point une fois pour toutes qui reproduit à l'échelle la loi hauteurdébit du Zêzere.

Le modèle fonctionne alors selon les lois de la similitude de Froude et reproduit à l'échelle les conditions d'écoulement dans les têtes amont et aval de la dérivation.

Comme dans tous les modèles analogues, il ne permet pas de reproduire à l'échelle la perte de charge par rugosité dans la galerie (qui n'obéit pas à la similitude de Froude). On peut toutefois mesurer la perte de charge correspondante se produisant sur le modèle et faire intervenir les résultats obtenus comme correctifs des mesures effectuées, en vue de déterminer la loi hauteurdébit de la galerie réelle. 


\section{IV. - ÉTUDES PRÉLIMINAIRES}

Le programme des travaux ayant prévu que le batardeau amont n'atteindrait sa hauteur définitive que plusieurs mois après la mise à sec du chantier et une crue d'une certaine importance pouvant survenir avant son achèvement, le principe du fonctionnement en charge de la galerie fut adopté. Il avait en effet l'avantage de mieux

Section de la galerie ...............

Rayon hydraulique .................

Longueur théorique de la galerie ..........

Longueur de la dérivation, y compris les têtes

Coefficient de Chezy évalué d'après la nature du revêtement et le rayon hydraulique de la galerie ..................

Cote de la ligne piézométrique vers la sortie pour le débit maximum ...........

(soit une récupération de $8,75 \mathrm{~m}$., le niveau aval correspondant étant $32,50 \mathrm{~m}$.) (1)

Vitesse d'écoulement dans la section courante pour $2.000 \mathrm{~m}^{3} / \mathrm{sec}$. . . . . . . . . . .

Energie cinétique correspondante ........

Perte de charge dans la galerie ..........

Evaluation de la perte de charge à l'entonne-

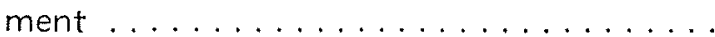

On en déduit la hauteur de retenue pour

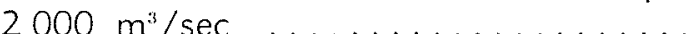

Dans cette phase préliminaire on a également déterminé, par une étude hydrodynamique graphique, la coupe longitudinale de la tête amont.

En effet, en raison des très grandes vitesses d'écoulement admises dans la galerie, il était nécessaire de choisir soigneusement le profil de ses formes d'entrée pour éviter tout décollement

(1) Une récupération nettement supérieure était hydrauliquement possible; toutefois, par suite de conditions d'exploitation particulières de la galerie, le maître de l'œuvre a renoncé au gain de premier établissement qu'aurait permis un abaissement supplémentaire de cet ouvrage. protéger le chantier, la galerie permettant ainsi d'écouler les crues moyennes avec une cote de retenue inférieure à celle qu'aurait nécessité une galerie de dérivation à surface libre.

Les essais ont été précédés d'une étude théorique assez poussée permettant de déterminer les caractéristiques principales suivantes de la galerie :

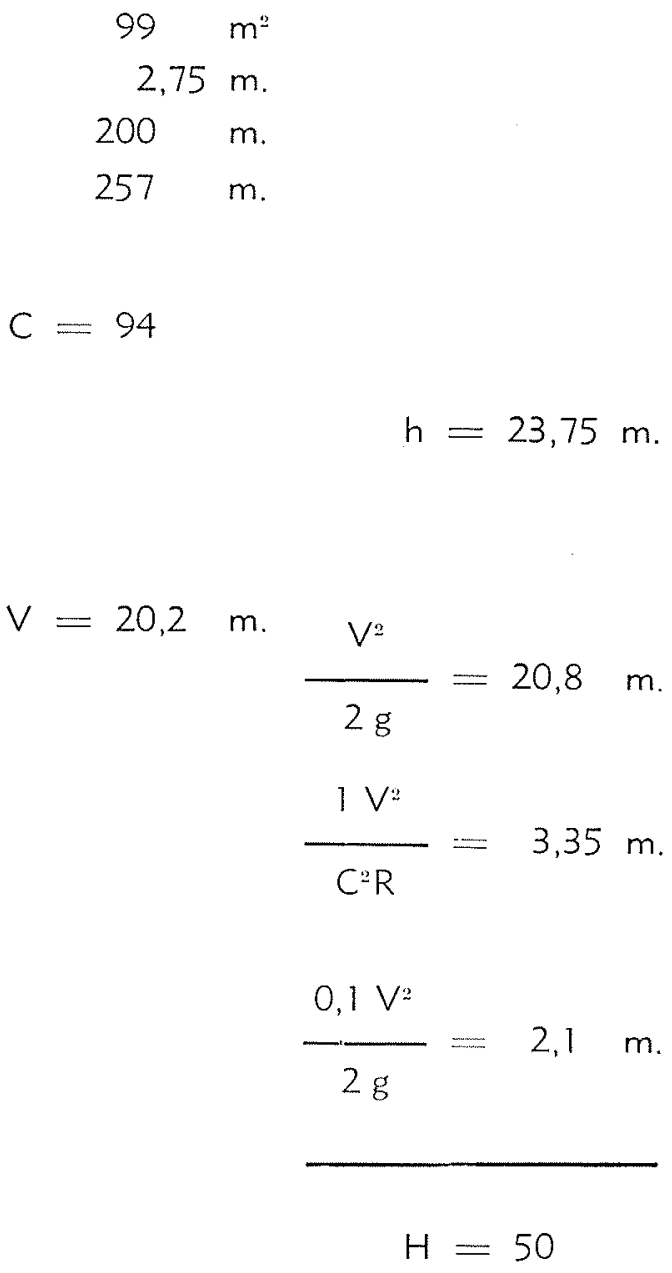

ou cavitation qui auraient provoqué des pertes de charge et des dégats importants.

Ces études préliminaires ont permis au maître de l'œuvre d'établir un avant-projet de la dériva.. tion lui permettant de commencer aussitôt les travaux de percement de la galerie et de commander les vannes et batardeaux qui devaient l'équiper, tandis que le modèle qui allait permettre d'approfondir l'étude des têtes de la dérivation entrait lui-même en construction. 


\section{DÉRIVATION PROVISOIRE}

Chambre du

PLAN DU MODELE RÉDUIT

\section{déversoir circulaire}

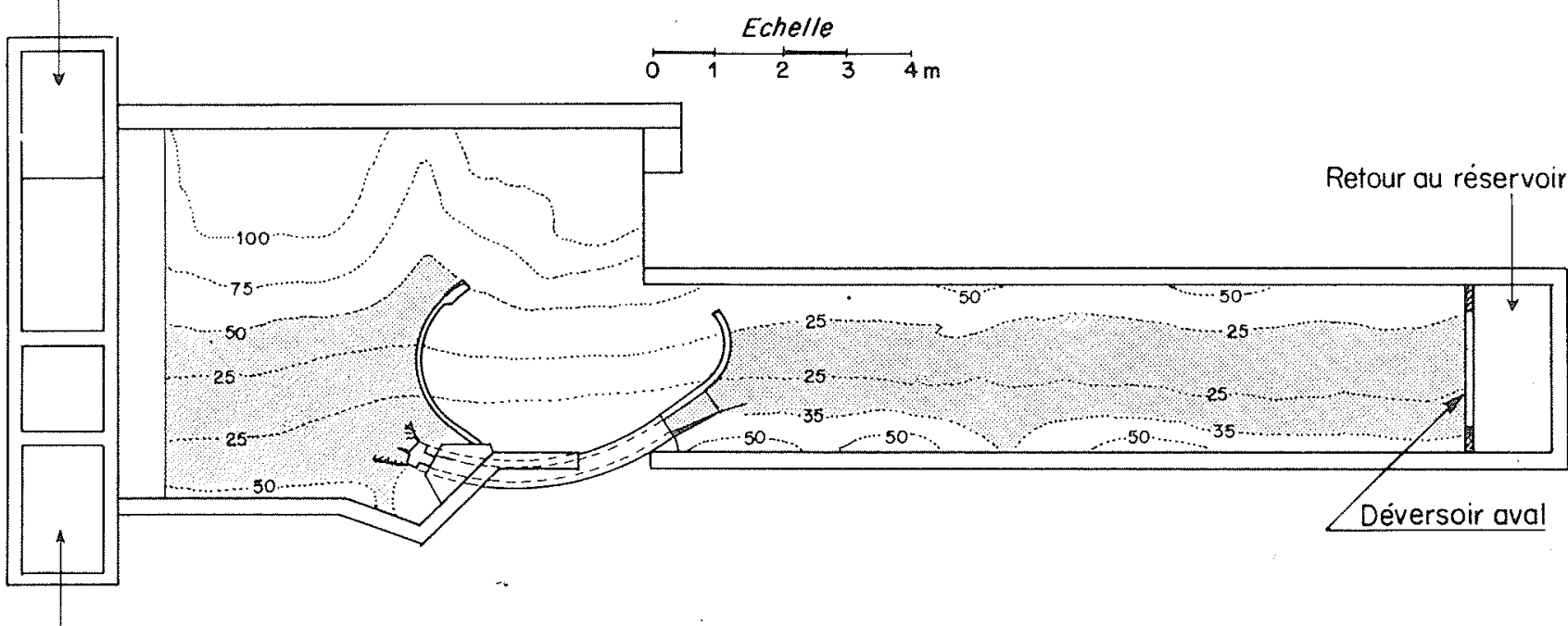

Chambre du

déversoir triangulaire

fig. .3

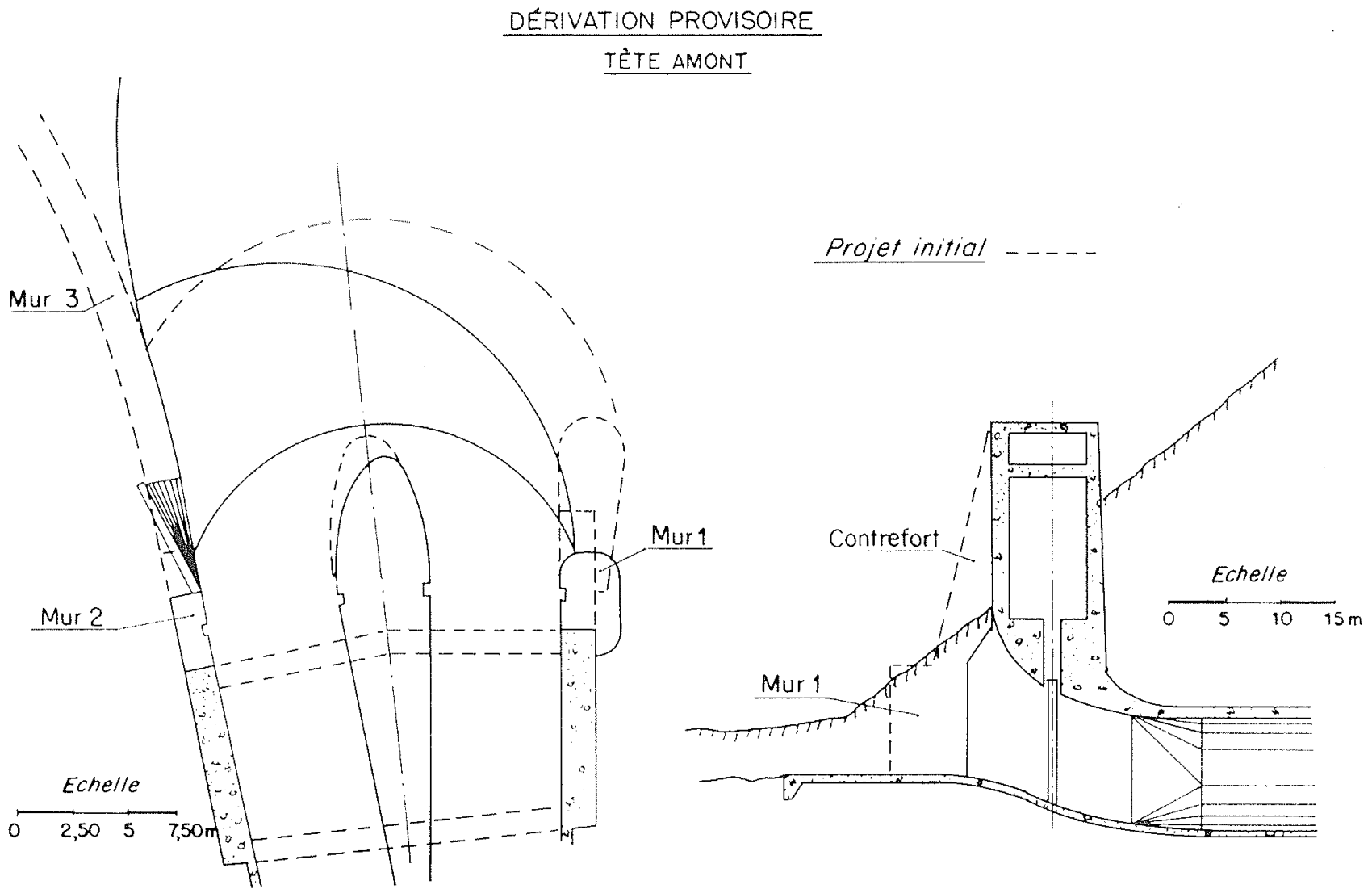

fig. 4 


\section{V. - LES ESSAIS}

\section{$1^{\circ}$ Essais de la tête amont.}

Dès les premiers essais de la tête amont, les mesures effectuées ont montré que les pertes de charge pour les formes déterminées préalablement par la méthode graphique étaient de l'ordre de grandeur de celles prévues dans les calculs préliminaires.

Toutefois, il était encore possible d'améliorer l'entonnement dans la galerie en adaptant mieux sa tête amont aux conditions d'alimentation imposées par la topographie locale, tout en cherchant à obtenir une certaine simplification du génie civil.

La figure 4 permet de comparer les formes prévues avant les essais avec celles définitive. ment proposées qui permettaient une diminution du volume de béton et des surfaces coffrées. ainsi qu'une réduction très sensible des déroche. ments à l'entrée de la galerie.

\section{$2^{\circ}$ Essais de la tête aval.}

Comme nous l'avons déjà indiqué, les ouvrages de la tête aval doivent permettre de récupérer une fraction de l'énergie cinétique de l'eau à la sortie de la galerie.

La galerie fonctionnant en charge, on pourrait penser tout d'abord que la récupération doive normalement être obtenue au moyen d'un divergent en charge. En fait, un tel ouvrage eût été mal adapté aux conditions locales, d'une part en raison de l'importante obliquité de la sortie par rapport aux lignes de plus grande pente du terrain et, d'autre part, la qualité médiocre du rocher au voisinage de la sortie rendant très onéreuse la construction d'un divergent de dimensions suffisantes.

Les études et essais ont permis par contre d'adopter un mode de récupération original assurant la possibilité d'établir une tête aval particulièrement bien adaptée aux conditions locales.

Le mode de fonctionnement de cet ouvrage pour les plus grosses crues est le suivant:

L'écoulement à la sortie de la galerie est fortement torrentiel (1).

(1) On a en effet, pour le débit maximum:

$$
F^{2}=\frac{\dot{V}^{2}}{g y}=5
$$

( $V$. g et y dés gnant respectivement la vitesse d'écoulement, l'ac$V$. g et y dés gnant respectivement la vitesse d'écoulement, l'ac-
célération de la pesanteur et la profondeur d'eau à l'entrée de la célération aval).
DÉRIVATION PROVISOIRE TETES AVAL AVEC RECUPERATION EN ECOULEMENT TORRENTIEL
0) - Avec bofoyers porolleles

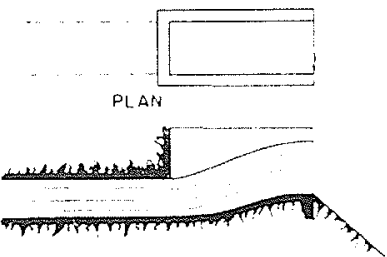

COUPE LONGITUDINALE b) Avec bopoyers divergents

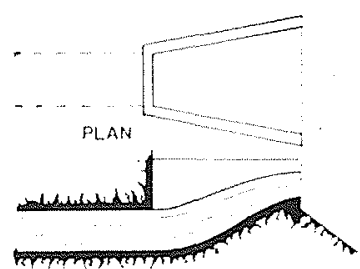

COUPE LONGITUOINALE fig. 5

Si la tête aval avait été constituée par deux bajoyers verticaux parallèles et un fond en contrepente (fig. 5 a), l'écoulement aurait ralenti progressivement dans cet ouvrage, et la hauteur d'eau aurait augmenté entre l'extrémité de la galerie et la fin de la tête aval. On obtiendrait donc ainsi une certaine récupération, mais celleci serait limitée, parce que le nombre de Froude diminuant rapidement le long de cette tête, le régime critique (section $\quad$ C. C.) serait bientôt atteint.

Faisons maintenant diverger les bajoyers (fig. $5 \mathrm{~b}$ ) et augmentons simultanément la contrepente afin de conserver dans chacune des sections de la tête aval la même surface mouillée que précédemment. Nous obtenons encore en C. C. la même récupération, mais la profondeur d'eau étant plus faible, le régime torrentiel subsiste et permet d'augmenter la récupération.

Dans ce cas, l'extrémité du radier est reportée à une cote relativement élevée, ce qui facilite les travaux d'asséchement du chantier correspondant.

C'est sur ce principe qu'a été basée l'étude de la tête aval dont le fonctionnement est illustré par les photographies voisines. Le seuil aval de cette tête est arasé à une cote à peine inférieure au niveau d'étiage du Zêzere, si bien que tout l'ouvrage a pu être construit sans établir de rideau de palplanches.

Comme on peut l'observer sur les photographies, la sortie de la galerie est fortement noyée pour les petites crues, puis se dénoie progressivement quand le débit augmente; on passe alors par un régime de forte turbulence qui disparait progressivement pour laisser place à une agitation de surface due à l'entraînement d'air lorsque la galerie est complètement dénoyée. 


\section{TÊTE AMONT}

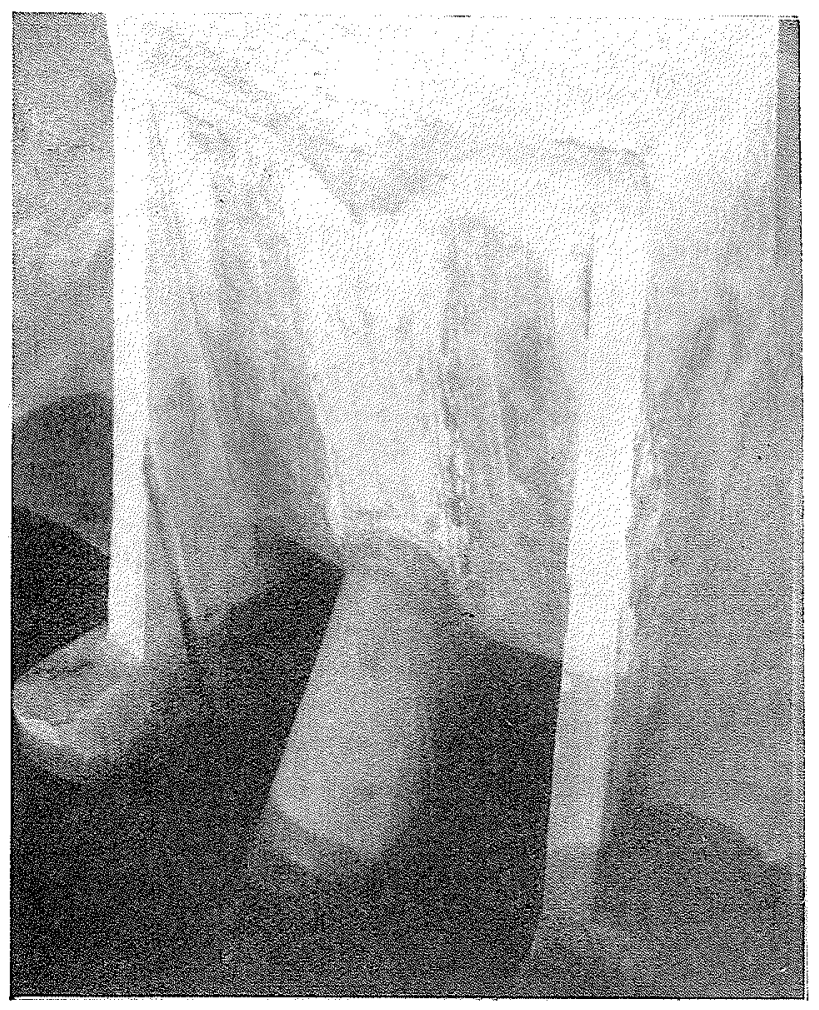

Forme initialement envisatée

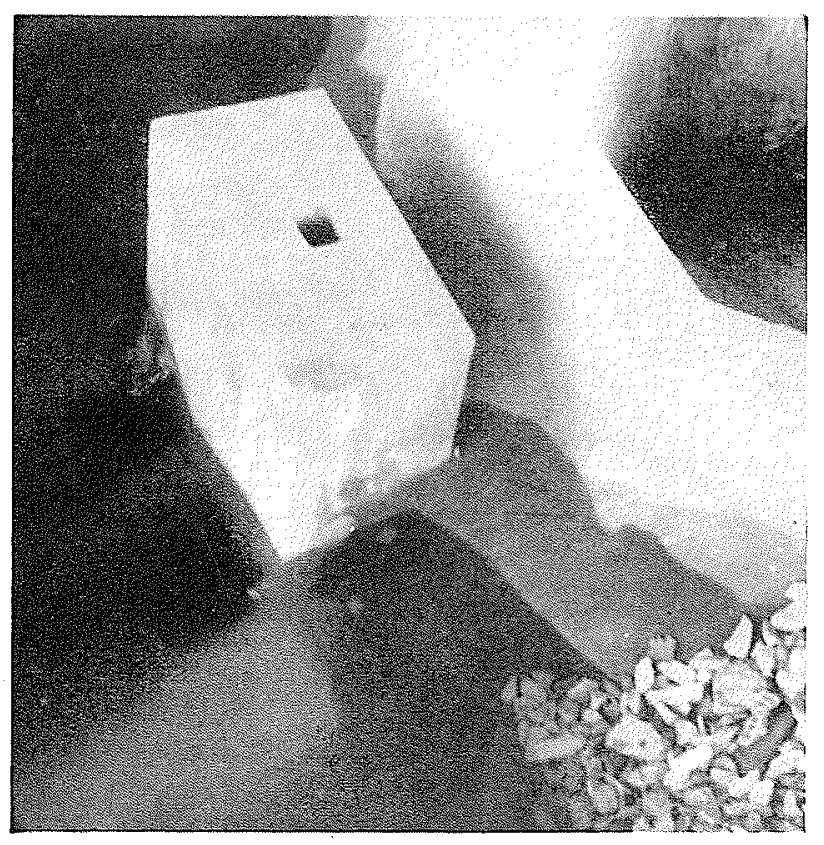

Entonnement d'un débit de $1.540 \mathrm{~m}^{3} / \mathrm{sec}$.

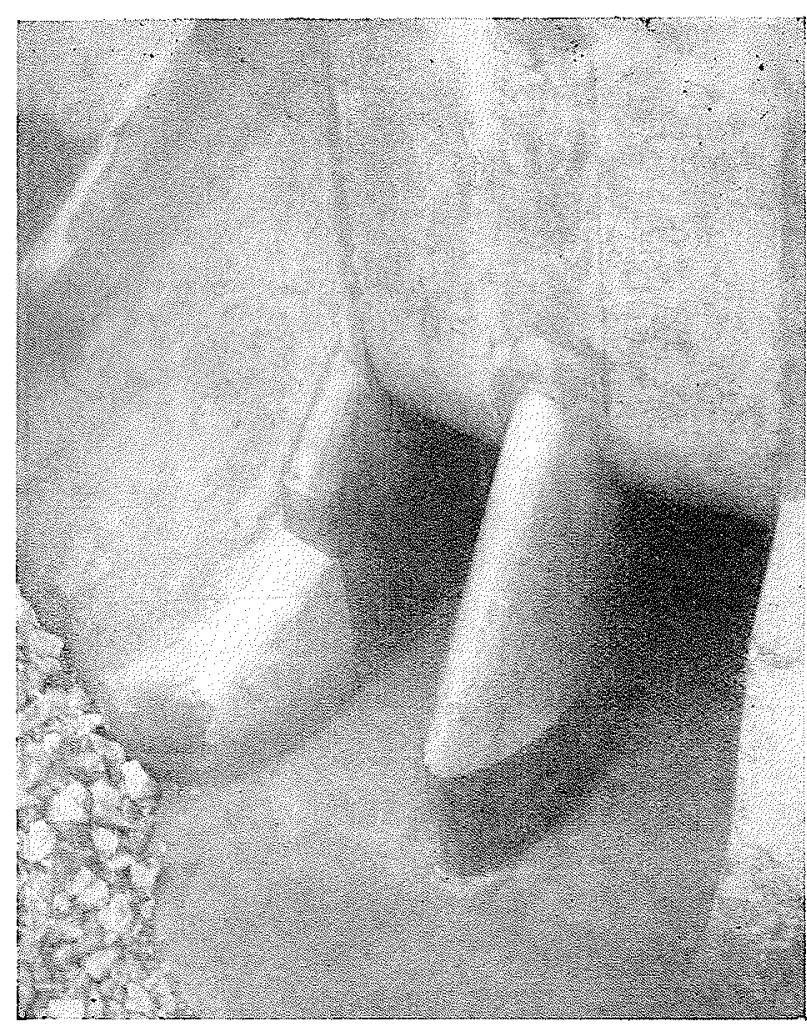

Forme réalisée après essai

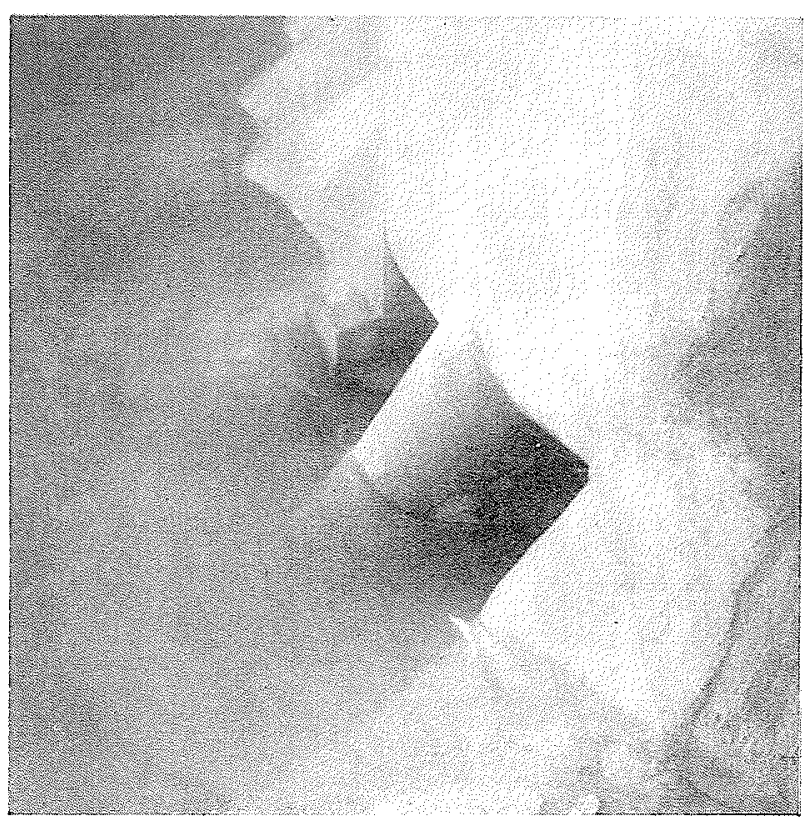

Entonnement d'un débit de $550 \mathrm{~m}^{3} / \mathrm{sec}$. 


\section{TÊTE AVAL}

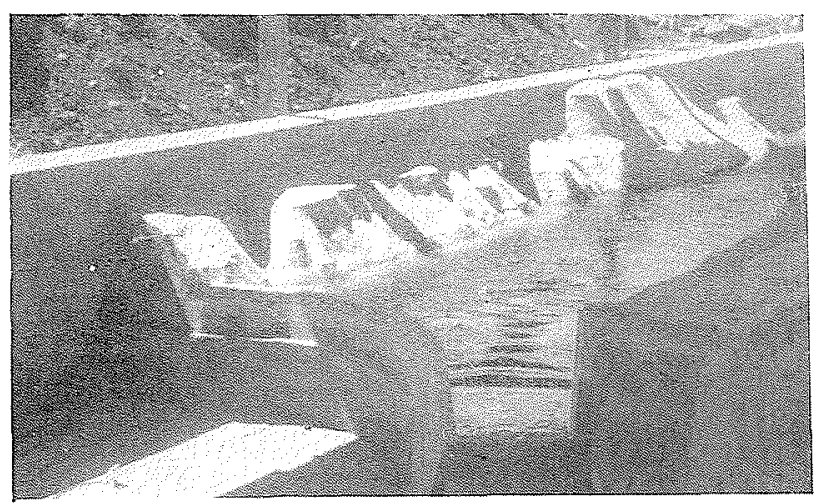

Débit $350 \mathrm{~m}^{7} / \mathrm{sec}$.

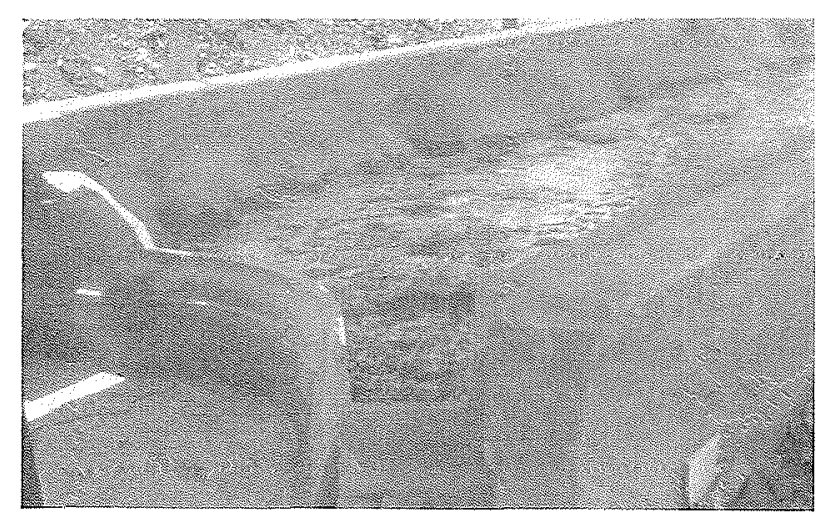

Débit $1.200 \mathrm{~m}^{5} / \mathrm{sec}$.

Observer l'agitation turbulente de la tête aval

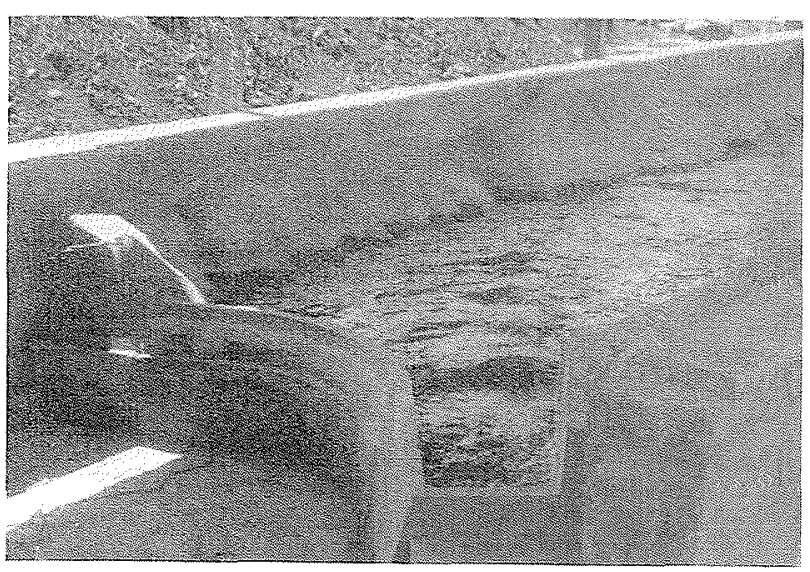

Débit $875 \mathrm{~m} / \mathrm{sec}$.

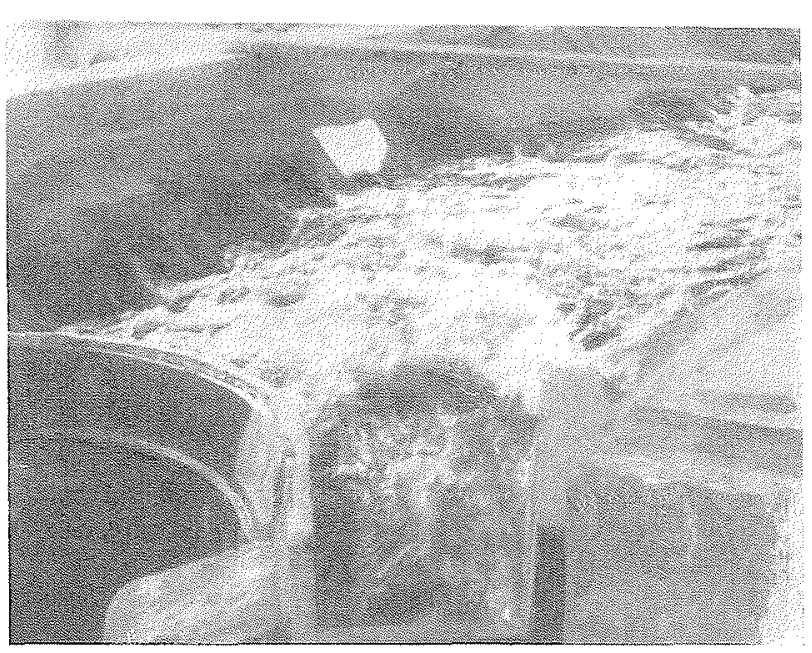

Débit $2.000 \mathrm{~m}^{3} / \mathrm{sec}$ 


\section{CRUE \\ DU 29 JANVIER 1948 (Débit $1.520 \mathrm{~m}^{3} / \mathrm{sec}$.)}

Le modelo $\rightarrow$

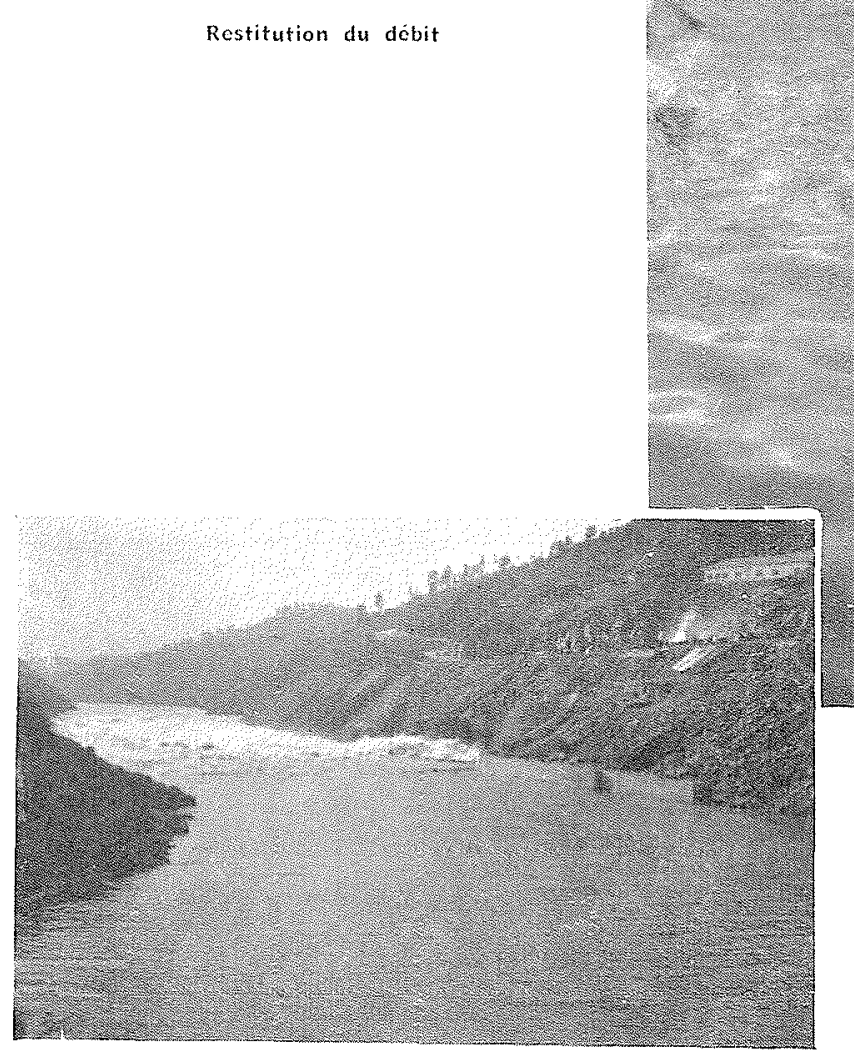

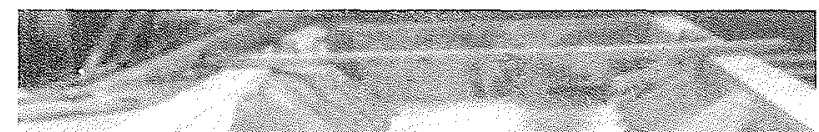

3.

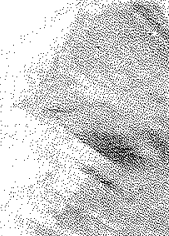

in?

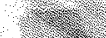

r.

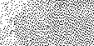

3.t.

- La réalité

Restitution du débi
Entonnement dans la galeric

(la tête amont n'est pas terminée)

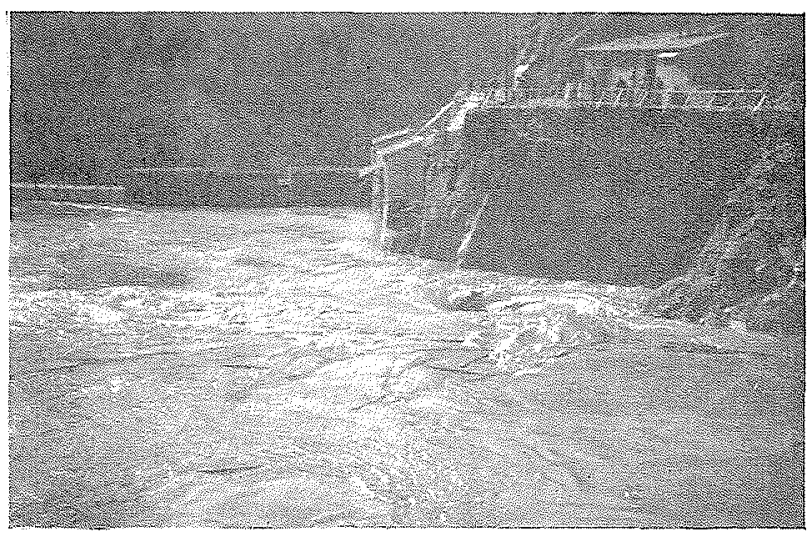




\title{
ECOULEMENT D'UN DÉBIT DE $1500 \mathrm{~m}^{3} / \mathrm{Sec}$.
}

\author{
ECOULEMENT DANS LA TÊTE AVAL
}

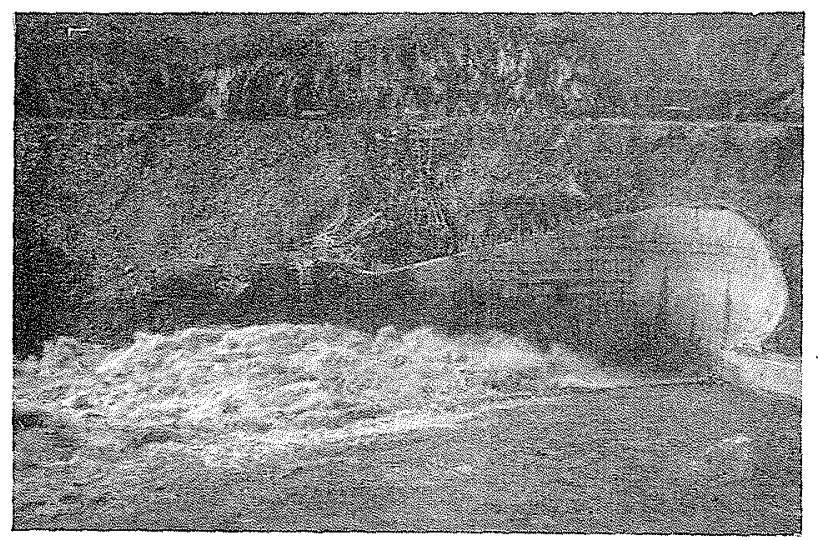

Dans la réalité

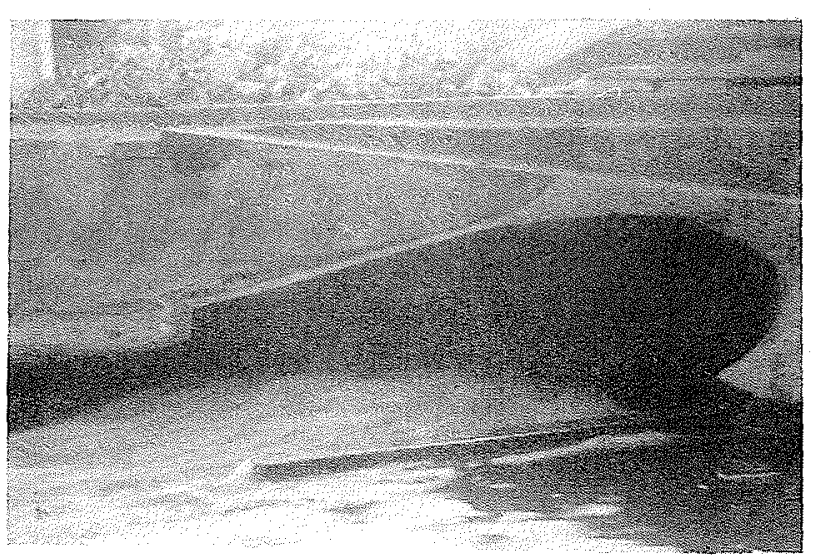

Sur le modèle

ECOULEMENT LE LONG DE LA RIVE GAUCHE DU ZÉZĖRE

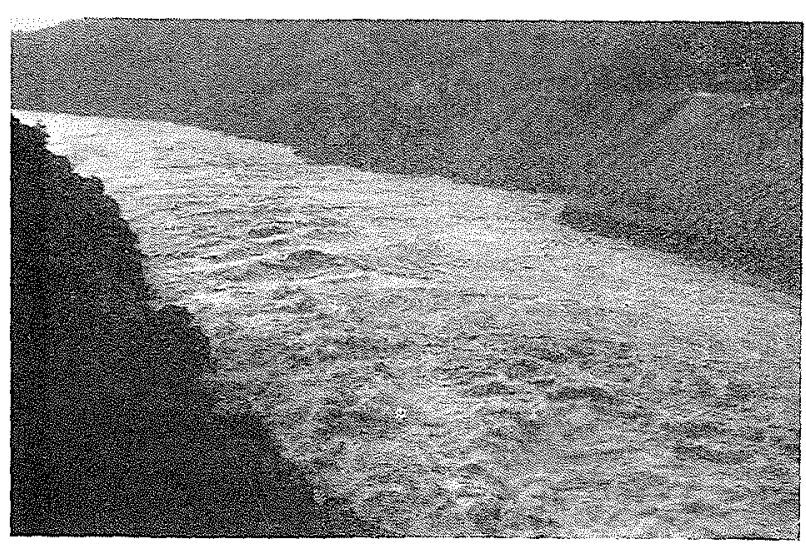

Dans la réalité

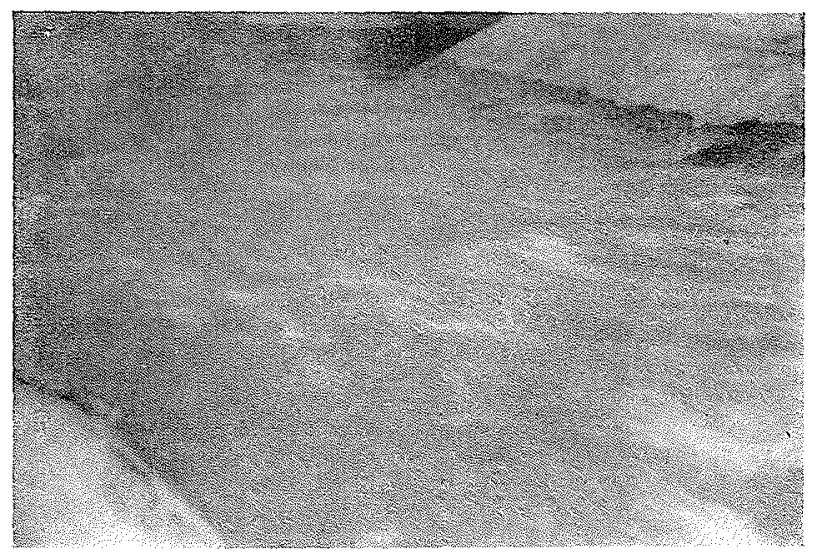

Sur le modèle 
La tête aval proprement dite ayant été mise au point, le modèle a permis d'étudier divers problèmes relatifs aux érosions et à la stabilité du batardeau aval soit en fonctionnement normal, soit en cas de déversement.

Enfin, au cours de l'avancement des travaux du chantier diverses particularités locales s'étant révélées, le modèle a encore permis de fournir au maître de l'œuvre des éléments d'appréciation lui permettant en dernière étape d'entreprendre l'adaptation définitive du projet de la dérivation et de réaliser, à la fois, des économies sensibles sur le temps d'exécution et sur le coût des ouvrages correspondants.

\section{VI. - FONCTIONNEMENT DE LA GALERIE DANS LA RÉALITÉ}

La dérivation de Castelo do Bode a été mise en eau en août 1947 et a donné passage depuis à un certain nombre de crues.

La plus importante de celles-ci a eu lieu le 29 janvier 1948. A cette époque, le batardeau amont était construit jusqu'à 7 mètres de sa cote définitive, tandis qu'il manquait un mètre au batardeau aval pour atteindre la cote du projet.

Pendant cette crue, le débit du Zêzere a atteint $1.900 \mathrm{~m}^{3} / \mathrm{sec}$., le niveau étant monté jusqu'à $75 \mathrm{~cm}$. de la cote d'arasement du batardeau amont ; le débit de la galerie s'est stabilisé pendant plusieurs heures à une valeur légèrement supérieure à $1.500 \mathrm{~m}^{3} / \mathrm{sec}$., tandis que la pointe de la crue était emmagasinée par la retenue.

Au cours de la montée de la crue, les débits pour lesquels s'établit la plus grande turbulence dans la tête aval, ont été atteints, puis largement dépassés, et cette turbulence - limitée d'ailleurs à la zone même de cet ouvrage - s'est effectivement révélée sans inconvénients.

Le lecteur pourra observer sur les photogra. phies l'écoulement de cette crue sur le modèle et dans la galerie réelle qui constitue, à notre connaissance, la dérivation la plus poussée construite jusqu'à maintenant.

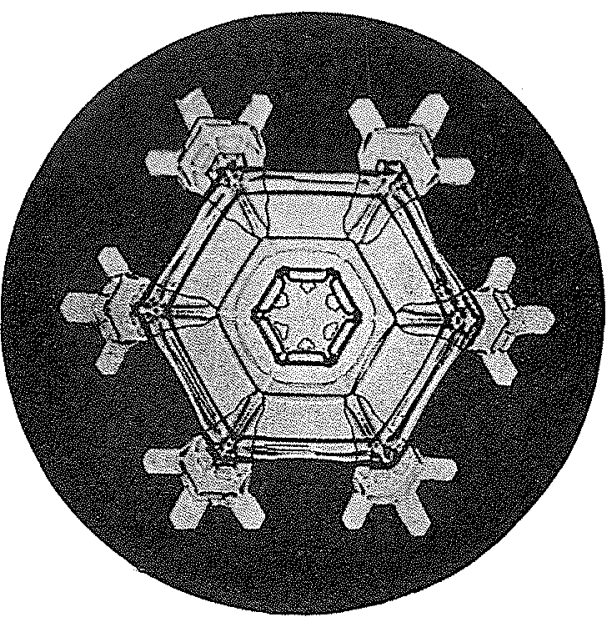

